\title{
Top quark polarization as a probe of charged Higgs bosons
}

\author{
Abdesslam Arhrib, ${ }^{1, *}$ Adil Jueid, ${ }^{2, \dagger}$ and Stefano Moretti ${ }^{3, *}$ \\ ${ }^{1}$ Département de Mathématiques, Faculté des Sciences et Techniques, \\ Université Abdelmalek Essaadi, B. 416, Tangier, Morocco \\ ${ }^{2}$ INPAC, Shanghai Key Laboratory for Particle Physics and Cosmology, \\ Department of Physics and Astronomy, Shanghai Jiao Tong University, Shanghai 200240, China \\ ${ }^{3}$ School of Physics and Astronomy, University of Southampton, Southampton, SO17 1BJ, United Kingdom
}

(Received 5 August 2018; published 5 December 2018)

\begin{abstract}
We study the production and decay of a heavy charged Higgs boson in the $b g \rightarrow t H^{-}$and $H^{-} \rightarrow b \bar{t}$ processes at the Large Hadron Collider (LHC). We show that the chiral structure of the $H^{-} t \bar{b}$ vertex entering both stages is sensitive to the underlying Higgs mechanism of electroweak symmetry breaking (EWSB) and we specifically demonstrate that one could distinguish between two popular realizations of a 2-Higgs doublet model (2HDM) embedding a new $H^{ \pm}$state, i.e., those with Type-I and -Y Yukawa couplings. The chiral structure of such a vertex, which is different in the two cases, in turn triggers a particular spin state of the top quark which is then transmitted to its decay products. Hence, both inclusive rates and exclusive observables can be used to extract the presence of such a charged Higgs boson state in LHC data.
\end{abstract}

DOI: 10.1103/PhysRevD.98.115006

\section{INTRODUCTION}

The top quark, discovered in the mid-1990s at the Tevatron by the D0 [1] and CDF [2] collaborations, is the heaviest elementary particle we know of. As such, it is the most sensitive probe of new physics beyond the standard model (BSM) onsetting at the $\mathrm{TeV}$ scale or above it. Further, due to its large mass, it can only be created as a real object in scattering processes taking place in the most recent particle accelerators like the now decommissioned Tevatron and the state-of-the-art Large Hadron Collider (LHC).

Thus, in recent years, studies of processes involving top quarks have become very numerous, also thanks to the large amount of data containing them which can be produced and analyzed. In this respect, an intriguing aspect is that the top quark has a very short lifetime and its decay width satisfies $\Gamma_{t} \simeq G_{F} m_{t}^{3} \gg \Lambda_{\mathrm{OCD}}^{2} / m_{t}$ implying that it decays immediately, i.e., before hadronization effects can take place. Thus, all its fundamental properties can be probed by studying its decay products (for a review, see [3-5]). Consequent measurements are therefore well

\footnotetext{
*aarhrib@gmail.com †adil.jueid@sjtu.edu.cn

*s.moretti@soton.ac.uk
}

Published by the American Physical Society under the terms of the Creative Commons Attribution 4.0 International license. Further distribution of this work must maintain attribution to the author(s) and the published article's title, journal citation, and DOI. Funded by SCOAP. established and can be used to either test the SM predictions or look for new physics beyond it.

One of the simplest SM extensions, embedding the now established Higgs mechanism of electroweak symmetry breaking (EWSB), is the 2-Higgs doublet model (2HDM), which was proposed four decades ago and has eventually found its way as a low energy manifestation of a higher scale fundamental dynamics which could be made manifest in both direct searches for the new Higgs boson states that it predicts, e.g., at the LHC, and indirect tests in flavor physics (for a theoretical and phenomenological review, see [6,7], respectively). In this scenario, two complex isodoublets are introduced to break the EW symmetry and eventually produce both fermion and gauge boson masses. The mediators of such mass generation dynamics are five Higgs boson states: two neutral $C P$-even ones $\left(h^{0}\right.$ and $H^{0}$, with $\left.m_{h^{0}}<m_{H^{0}}\right)$, one $C P$ odd neutral one $\left(A^{0}\right)$ and a pair of charged ones $\left(H^{ \pm}\right)$.

The top quark couples to all of these, hence, it is no surprise that top quark processes have been studied extensively within the $2 \mathrm{HDM}$, not least because the structure of the (Yukawa) coupling between such a fermion and (pseudo)scalar bosons can reveal the properties of the underlying $2 \mathrm{HDM}$. Such studies concerned not only inclusive production cross sections [8-10] but also the possibility to exclusively extract the aforementioned couplings [11-14], using the leading SM-like decay channel of the top quark as well as its rare and some exotic ones [15]. In essence, it was indeed proven that the top quark can serve as a discovery probe of the $2 \mathrm{HDM}$ and also to discriminate between its different realizations. 
It is the purpose of this paper to contribute to this endeavor by exploiting polarization effects of the top quark, including $t \bar{t}$ spin correlations (see, e.g., [16-18] and references therein), in studies of $2 \mathrm{HDM}$ charged Higgs bosons at the LHC, for the case when $m_{H^{ \pm}}>m_{t}$, i.e., of a heavy $H^{ \pm}$state. By building upon earlier results [13], which postulated a $2 \mathrm{HDM}$ Type-II structure (henceforth, 2HDM-II), we complement these by showing that other 2HDM paradigms can similarly be probed, e.g., the $2 \mathrm{HDM}$ Type-I (henceforth, 2HDM-I) and 2HDM Type-Y (henceforth, $2 \mathrm{HDM}-\mathrm{Y}$ or flipped). Furthermore, we will investigate a larger variety of experimental observables that can be used for the above purpose. We will eventually show that the latter can profitably be exploited both as a mean to improve sensitivity of current heavy $H^{ \pm}$searches and as a post-discovery tool to characterize the extracted signals in terms of the underlying $2 \mathrm{HDM}$. The underpinning element in this study is the chiral structure of the $H^{-} t \bar{b}$ vertex and how it affects the production and decay of a heavy charged Higgs boson in the $b g \rightarrow t H^{-}$and $H^{-} \rightarrow b \bar{t}$ processes at the CERN machine.

The plan of this paper is as follows. In the next section we describe the $2 \mathrm{HDM}$ as well as define benchmark points in its parameter space amenable to phenomenological investigation. We then define observables that can be used in experimental studies. After presenting our numerical results, we conclude.

\section{MODEL, CONSTRAINTS AND BENCHMARK POINTS}

\section{A. The model}

In this section, we discuss briefly the $2 \mathrm{HDM}$ and several constraints imposed on its parameter space. In this model, as already mentioned, two scalar isodoublets are included to break the EW gauge symmetry and give rise to fermion and gauge boson masses. However, due to the presence of two Higgs doublets $\left(\Phi_{1}\right.$ and $\left.\Phi_{2}\right)$, absence of large tree level flavor changing neutral currents (FCNCs) is not guaranteed unless a discrete symmetry, $Z_{2}$, either exact or sofltly broken, is imposed [19]. Under this symmetry, (pseudo) scalar fields transform as $\Phi_{1} \rightarrow \Phi_{1}$ and $\Phi_{2} \rightarrow-\Phi_{2}$, hence, four possible combinations of (pseudo)scalar and fermion interactions are possible, which give rise to four possible types of $2 \mathrm{HDM}$ (see, e.g., [20] for more details). Hereafter, we define the $2 \mathrm{HDM}$-I the model where only $\Phi_{2}$ couples to all the fermions exactly as in the SM while the 2HDM Type-II (henceforth, 2HDM-II) is defined such that $\Phi_{2}$ couples to up-type quarks and $\Phi_{1}$ to down-type quarks and charged leptons. In the $2 \mathrm{HDM}$ type-X (2HDM$\mathrm{X}$, called also lepton-specific), the charged leptons couple to $\Phi_{1}$ while all the quarks couple to $\Phi_{2}$. Finally, the $2 \mathrm{HDM}$ $\mathrm{Y}$ case is instead built such that $\Phi_{2}$ couples to up-type quarks and leptons and $\Phi_{1}$ couples to down-type quarks.
The Lagrangian representing the Yukawa interactions is given by:

$$
\begin{aligned}
-\mathcal{L}_{\text {Yukawa }}= & \bar{Q}_{L, i} Y_{u, i j} \tilde{\Phi}_{2} u_{R, j}+\bar{Q}_{L, i} Y_{d, i j} \Phi_{d} d_{R, j} \\
& +\bar{L}_{L} Y_{\ell, i j} \Phi_{\ell} \ell_{R, j}+\text { H.c. },
\end{aligned}
$$

where $\Phi_{\alpha}, \alpha=\ell, d$ is either $\Phi_{1}$ or $\Phi_{2}, Y_{\alpha, i j}$ is a set of $3 \times 3$ Yukawa matrices and $i, j=1,2,3$ are the generation indices.

The most general gauge-invariant, renormalizable and $C P$-conserving scalar potential, with a softly broken $Z_{2}$ symmetry, is given by:

$$
\begin{aligned}
V\left(\Phi_{1}, \Phi_{2}\right)= & \mu_{11}^{2}\left|\Phi_{1}\right|^{2}+\mu_{22}^{2}\left|\Phi_{2}\right|^{2}-\mu_{12}^{2}\left(\Phi_{1}^{\dagger} \Phi_{2}+\Phi_{2}^{\dagger} \Phi_{1}\right) \\
& +\lambda_{1}\left|\Phi_{1}\right|^{4}+\lambda_{2}\left|\Phi_{2}\right|^{4}+\lambda_{3}\left|\Phi_{1}\right|^{2}\left|\Phi_{2}\right|^{2} \\
& +\lambda_{4}\left|\Phi_{1}^{\dagger} \Phi_{2}\right|^{2}+\frac{\lambda_{5}}{2}\left[\left(\Phi_{1}^{\dagger} \Phi_{2}\right)^{2}+\text { H.c. }\right] .
\end{aligned}
$$

The parameters $\mu_{11,22}^{2}, \lambda_{i},(i=1 \ldots 4)$ are real valued while the parameters $\mu_{12}^{2}$ and $\lambda_{5}$ could be complex valued in the case of $C P$-violation. The two Higgs doublets $\Phi_{1}$ and $\Phi_{2}$ are parametrized as follows:

$$
\Phi_{i}=\left(\begin{array}{c}
\phi_{i}^{+} \\
v_{i}+\frac{1}{\sqrt{2}}\left(h_{i}+i \omega_{i}\right)
\end{array}\right), \quad i=1,2
$$

where $v_{1}$ and $v_{2}$ are the vacuum expectation values (VEVs) of the two Higgs doublets. After EWSB, three degrees of freedom are absorbed by the longitudinal gauge bosons $W^{ \pm}, Z^{0}$ and we are left with the aforementioned five (pseudo)scalar degrees of freedom as real massive particles: $h^{0}, H^{0}, A^{0}$ and $H^{ \pm}$. These mass eigenstates are obtained through the following relations:

$$
\begin{aligned}
& \left(\begin{array}{l}
h_{1} \\
h_{2}
\end{array}\right)=R(\alpha)\left(\begin{array}{c}
H^{0} \\
h^{0}
\end{array}\right), \quad\left(\begin{array}{l}
\phi_{1}^{ \pm} \\
\phi_{2}^{ \pm}
\end{array}\right)=R(\beta)\left(\begin{array}{l}
G^{ \pm} \\
H^{ \pm}
\end{array}\right), \\
& \left(\begin{array}{l}
\omega_{1} \\
\omega_{2}
\end{array}\right)=R(\beta)\left(\begin{array}{c}
G^{0} \\
A^{0}
\end{array}\right),
\end{aligned}
$$

where $R(\theta)$ is an orthogonal matrix defined as

$$
R(\theta)=\left(\begin{array}{rr}
\cos \theta & -\sin \theta \\
\sin \theta & \cos \theta
\end{array}\right),
$$

with $\beta$ defined via $\tan \beta=v_{2} / v_{1}$ and $\alpha$ the mixing angle between the $C P$-even interaction states $h_{i}(i=1,2)$. The $\omega_{i}$ and $\phi_{i}^{ \pm}(i=1,2)$ interaction states give in turn rise to the $C P$-odd Higgs, charged Higgs and Goldstone modes.

In terms of Higgs mass eigenstates, from the Yukawa Lagrangian in Eq. (1) we get 


$$
\begin{aligned}
-\mathcal{L}_{\text {Yukawa }}= & \sum_{\psi=u, d, \ell}\left(\frac{m_{\psi}}{v} \kappa_{\psi}^{h} \bar{\psi} \psi h^{0}+\frac{m_{\psi}}{v} \kappa_{\psi}^{H} \bar{\psi} \psi H^{0}-i \frac{m_{\psi}}{v} \kappa_{\psi}^{A} \bar{\psi} \gamma_{5} \psi A^{0}\right) \\
& +\left(\frac{V_{u d}}{\sqrt{2} v} \bar{u}\left(m_{u} \kappa_{u}^{A} P_{L}+m_{d} \kappa_{d}^{A} P_{R}\right) d H^{+}+\frac{m_{\ell} \kappa_{\ell}^{A}}{\sqrt{2} v} \bar{\nu}_{L} \ell_{R} H^{+}+\text {H.c. }\right),
\end{aligned}
$$

where the $\kappa_{i}$ 's are the Yukawa couplings in the $2 \mathrm{HDM}, V_{u d}$ is a Cabibbo-Kobayashi-Maskawa (CKM) matrix element while $P_{L / R}=\left(1-/+\gamma_{5}\right) / 2$ are the Left (L) and Right (R) chiral projectors. We give in Table $\mathrm{I}$ the values of the couplings in the four types of Yukawa interactions of the 2HDM. From this, it is clear that, as far as only the charged Higgs couplings to quarks are concerned, there is no difference between 2HDM-I and 2HDM-X or between 2HDM-II and 2HDM-Y.

Finally, note that the Higgs sector of the 2HDM has 7 independent parameters, which can be $\operatorname{taken}$ as $\tan \beta$, $\sin (\beta-\alpha), \mu_{12}^{2}$ and the four physical Higgs masses.

\section{B. Constraints and benchmark points}

The experimental data collected so far at the LHC regarding the $125 \mathrm{GeV}$ Higgs boson discovered in 2012 seem to indicate that the couplings of such a particle to SM objects are to a large extent similar to those in the SM, hence such a Higgs state is SM-like. Therefore, any BSM physics must contain a SM-like Higgs boson. This puts severe constraints on the parameter space of the various 2HDM types and mostly (though not always) pushes the generic model close to its decoupling limit where $h^{0}$ mimics the SM-like Higgs boson and the other states are rather heavy. The decoupling limit is characterized by $\sin (\beta-\alpha) \approx 1(\beta-\alpha \approx \pi / 2)$ and $m_{H^{0}}, m_{A^{0}}, m_{H \pm} \gg m_{Z^{0}}$, $m_{W^{ \pm}}[21]$.

In our analysis, we indeed require that the lightest $C P$-even scalar is the observed Higgs boson with $m_{h^{0}}=$ $125 \mathrm{GeV}$ and assume that $\beta-\alpha \approx \pi / 2$, which automatically implies that $h^{0}$ couplings to SM particles are SM-like. The other (pseudo)scalars are chosen nearly degenerate in order to avoid constraints from EW precision measurements. At any rate, all the parameters of the model are subject to several theoretical and experimental constraints. The benchmark points that we will use in our study are selected in such a way that they satisfy all of these. As we are concerned with a charged Higgs state, we start by dwelling at some length on the most relevant constraints on its mass and couplings.

The charged Higgs mass is subject to a number of constraints from several $B$-physics observables as well as from direct experimental searches at the LHC (and previous colliders). The most stringent flavor bound comes from the $B$-meson branching ratios (BRs), chiefly, $\operatorname{BR}\left(B \rightarrow X_{s} \gamma\right)$ [22]. For instance, in the 2HDM-II, the current measurement of $\mathrm{BR}\left(B \rightarrow X_{s} \gamma\right)$ forces the charged Higgs boson mass to be larger than about $580 \mathrm{GeV}$ [22] (for any $\tan \beta$ ) while in the 2 HDM-I one can still obtain a $H^{ \pm}$with a mass as low as $100-200 \mathrm{GeV}$ provided that $\tan \beta \geq 2$. Note that, in 2 HDM-II and -Y, if we allow $b \rightarrow s \gamma$ measurements at the $3 \sigma$ level, then it would imply a reduction on the charged Higgs bound from $580 \mathrm{GeV}$ down to $440 \mathrm{GeV}$ [23].

From the direct search side, the combined void searches from all four LEP collaborations imply the lower limit $m_{H^{ \pm}}>78.6 \mathrm{GeV}$ at $95 \%$ Confidence Level (CL), which applies to all models in which $\operatorname{BR}\left(H^{ \pm} \rightarrow \tau \nu\right)+$ $\operatorname{BR}\left(H^{ \pm} \rightarrow c s\right)=1$ [24]. Searches for a light charged Higgs boson at the Tevatron and LHC have instead been performed from top quark decays: $t \rightarrow b H^{ \pm}$followed by $H^{ \pm} \rightarrow \tau \nu$ or $H^{ \pm} \rightarrow c s$. A search for heavy charged Higgs states has also been conducted through $p p \rightarrow t H^{ \pm}+X$ with $H^{ \pm} \rightarrow \tau \nu$. For light $H^{ \pm}$states, the ATLAS and CMS experiments have already drawn an exclusion on $\mathrm{BR}\left(t \rightarrow b H^{ \pm}\right) \times \mathrm{BR}\left(H^{ \pm} \rightarrow \tau \nu\right)[7,25,26]$. In the context of some specific minimal supersymmetric standard model (MSSM) scenarios, these results exclude nearly all $\tan \beta \geq 1$ and $H^{ \pm}$masses in the range $80-160 \mathrm{GeV}$. For a heavy $H^{ \pm}$, they exclude a region of parameter space with high $\tan \beta$ for $H^{ \pm}$masses between 200 and $250 \mathrm{GeV}[25,26]$.

However, one need to keep in mind the following.

(i) In the $2 \mathrm{HDM}$ framework, the above limits, which are based on fermionic decays of the charged Higgs state, can be weakened if any of the bosonic decays $H^{ \pm} \rightarrow W^{ \pm} h^{0}$ and/or $H^{ \pm} \rightarrow W^{ \pm} A^{0}$ are open [27,28].

TABLE I. Yukawa couplings in terms of mixing angles in the 2HDM-I, -II, -X and -Y.

\begin{tabular}{lccccccccc}
\hline \hline & $\kappa_{u}^{h}$ & $\kappa_{d}^{h}$ & $\kappa_{\ell}^{h}$ & $\kappa_{u}^{H}$ & $\kappa_{d}^{H}$ & $\kappa_{\ell}^{H}$ & $\kappa_{u}^{A}$ & $\kappa_{d}^{A}$ & $\kappa_{\ell}^{A}$ \\
\hline Type-I & $c_{\alpha} / s_{\beta}$ & $c_{\alpha} / s_{\beta}$ & $c_{\alpha} / s_{\beta}$ & $s_{\alpha} / s_{\beta}$ & $s_{\alpha} / s_{\beta}$ & $s_{\alpha} / s_{\beta}$ & $\cot \beta$ & $-\cot \beta$ & $-\cot \beta$ \\
Type-II & $c_{\alpha} / s_{\beta}$ & $-s_{\alpha} / c_{\beta}$ & $-s_{\alpha} / c_{\beta}$ & $s_{\alpha} / s_{\beta}$ & $c_{\alpha} / c_{\beta}$ & $c_{\alpha} / c_{\beta}$ & $\cot \beta$ & $\tan \beta$ & $\tan \beta$ \\
Type-X & $c_{\alpha} / s_{\beta}$ & $c_{\alpha} / s_{\beta}$ & $-s_{\alpha} / c_{\beta}$ & $s_{\alpha} / s_{\beta}$ & $s_{\alpha} / s_{\beta}$ & $c_{\alpha} / c_{\beta}$ & $\cot \beta$ & $-\cot \beta$ & $\tan \beta$ \\
Type-Y & $c_{\alpha} / s_{\beta}$ & $-s_{\alpha} / c_{\beta}$ & $c_{\alpha} / s_{\beta}$ & $s_{\alpha} / s_{\beta}$ & $c_{\alpha} / c_{\beta}$ & $s_{\alpha} / s_{\beta}$ & $\cot \beta$ & $\tan \beta$ & $-\cot \beta$ \\
\hline \hline
\end{tabular}


(ii) Limits on charged Higgs bosons decaying to $\tau \nu$ final states valid for the $2 \mathrm{HDM}$-II might be invalidated in the framework of the 2HDM-Y where the $H^{ \pm} \rightarrow \tau \nu$ decay rate behaves like $1 / \tan \beta$ for large $\tan \beta$. This also applies to heavy $H^{0} / A^{0}$ searches in the $\tau^{+} \tau^{-}$ channel because also the $H^{0} \tau^{+} \tau^{-}$and $A^{0} \tau^{+} \tau^{-}$ couplings are proportional to $1 / \tan \beta$ for large $\tan \beta$ [29]. A detailed analysis of the status of charged Higgs in the four types $2 \mathrm{HDM}$ can be found in [30].

As intimated, in our scenario, we will assume that $H^{0}, A^{0}$ and $H^{ \pm}$states are nearly degenerate in mass so that $H^{ \pm} \rightarrow$ $W^{ \pm} H^{0} / W^{ \pm} A^{0}$ decays are closed. Furthermore, since the coupling $H^{ \pm} W^{ \pm} h^{0}$ is proportional to $\cos (\beta-\alpha)$, which is very small in our $2 \mathrm{HDM}$ scenarios, the decay $H^{ \pm} \rightarrow W^{ \pm} h^{0}$ will be suppressed. Therefore, the decays $H^{ \pm} \rightarrow t b$ and $H^{ \pm} \rightarrow \tau \nu$ proceeds with a almost $100 \%$ cumulative BR.

We have used the public code $2 \mathrm{HDMC}$ [31] to make a scan on $\mu_{12}^{2}, m_{H^{ \pm}}, \tan \beta, \sin (\beta-\alpha)$ and the Higgs masses (with the boundary condition that $m_{h^{0}}=125 \mathrm{GeV}$ ). The code also allows for the calculation of all relevant charged Higgs BRs and checks several theoretical constraints such as boundedness from below of the scalar potential, tree level perturbative unitarity as well as the EW precision observables $S$ and $T$. Regarding the latter, the aforementioned requirement of near mass degeneracy amongst $H^{0}$, $A^{0}$ and $H^{ \pm}$(specifically, in our scan, that $m_{H^{0}}=$ $m_{H^{ \pm}}+4 \mathrm{GeV}=m_{A^{0}}+2 \mathrm{GeV}$ ), implemented in order to suppress $H^{ \pm} \rightarrow W^{ \pm} H^{0} / W^{ \pm} A^{0}$ decays, turns out to be useful to also evade $T$ parameter limits, which can easily be achieved whenever $m_{H^{ \pm}} \approx m_{A^{0}}$.

The compatibility of the other $h^{0}$ properties with those of the observed SM-like Higgs boson has been checked using HigGSSIGNALS [32] through a $\chi^{2}$ minimization of the Higgs boson signal strengths. Constraints from void searches for

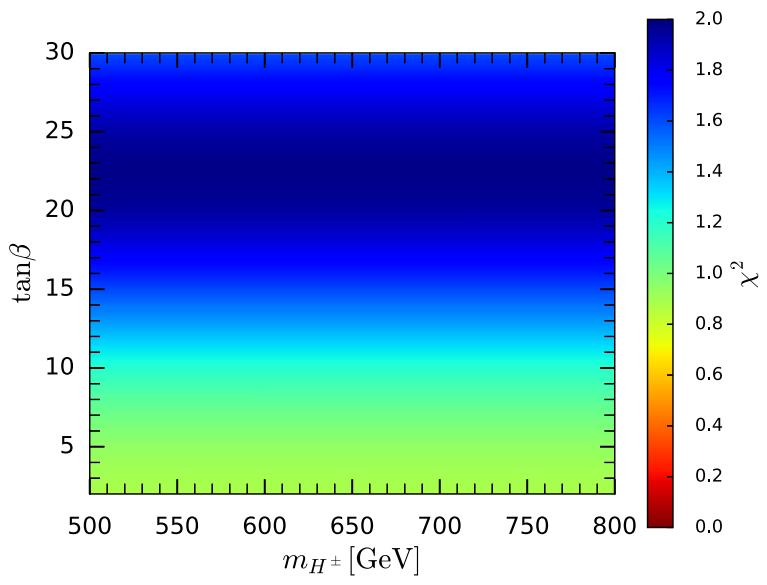

extra Higgs bosons at LEP, Tevatron and the LHC have been applied using HiggsBounds [33]. Herein, a parameter point is excluded at $95 \% \mathrm{CL}$ if the ratio $r_{95 \%}$ between model predictions and data, defined by

$$
r_{95 \%}=\frac{S_{\text {model }}}{S_{\text {observed }}^{95 \%}}
$$

is larger than 1.

In Fig. 1, we show a scatter plot on the $\left(\tan \beta, m_{H^{ \pm}}\right)$plane illustrating the impact of experimental constraints on, e.g., the 2HDM-II. In the left panel we show the $\chi^{2}$ behavior while in the right panel we show the $r_{95 \%}$ one. From the first plot, we can see clearly that the SM-like Higgs boson measurements are consistent with 2HDM-II at the $2 \sigma$ level whereas, from the second plot, we can deduce that the absence of discovery of additional (pseudo)scalars exclude $\tan \beta>8$ for all the charged Higgs masses at 95\% CL. Here, the strongest constraint comes from $g g \rightarrow A^{0} / H^{0} \rightarrow$ $\tau^{+} \tau^{-}$since this process in the 2 HDM-IIis $\tan \beta$ enhanced. As for the 2HDM-I, the effect of the Higgs signal strength measurements is similar to that of the 2 HDM-II. However, the effect of void searches for additional Higgs states does not exclude any points in this case, since the $A^{0} / H^{0} \rightarrow$ $\tau^{-} \tau^{+}$decay rates are proportional to $1 / \tan \beta$, as remarked upon already. Also in the case of the 2 HDM-Y such a $1 / \tan \beta$ dependence enables large $\tan \beta$ values, up to 50 or so, so long that $H^{ \pm}$is heavy enough. Therefore, in the mass region of interest, $m_{H^{ \pm}}>m_{t}+m_{b} \approx 180 \mathrm{GeV}$, where $H^{ \pm} \rightarrow t b$ decays are dominant, both the 2HDM-I and -Y offer considerable regions of available parameter space with the notable difference (which we while exploit in the remainder) that the former can attain small $\tan \beta$ values that are instead precluded to the latter.

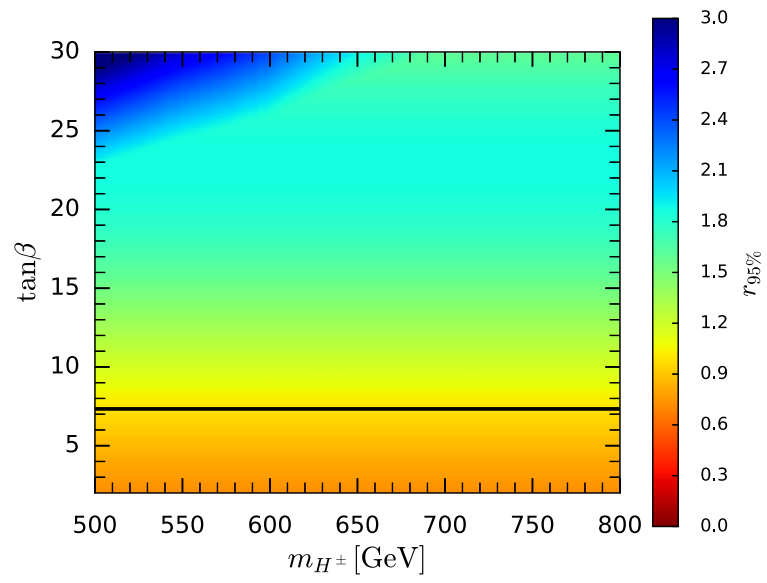

FIG. 1. Left: Scatter plot of $\tan \beta$ and $m_{H}^{ \pm}$showing the constraints from Higgs boson signal strengths and mass measurements using HigGSSignAls. Right: The effect of the void direct searches for additional (pseudo)scalars on $\tan \beta$ and $m_{H^{ \pm}}$using HigGsBounds. The $\chi^{2}$ (from HigGsSignALS) and $r_{95 \%}$ (from HigGsBounds) values are shown as a color map. The horizontal black line on the right shows $r_{95 \%}=1$ above which the parameter point is excluded at $95 \%$ CL. We illustrate here the case of the 2HDM-II. 

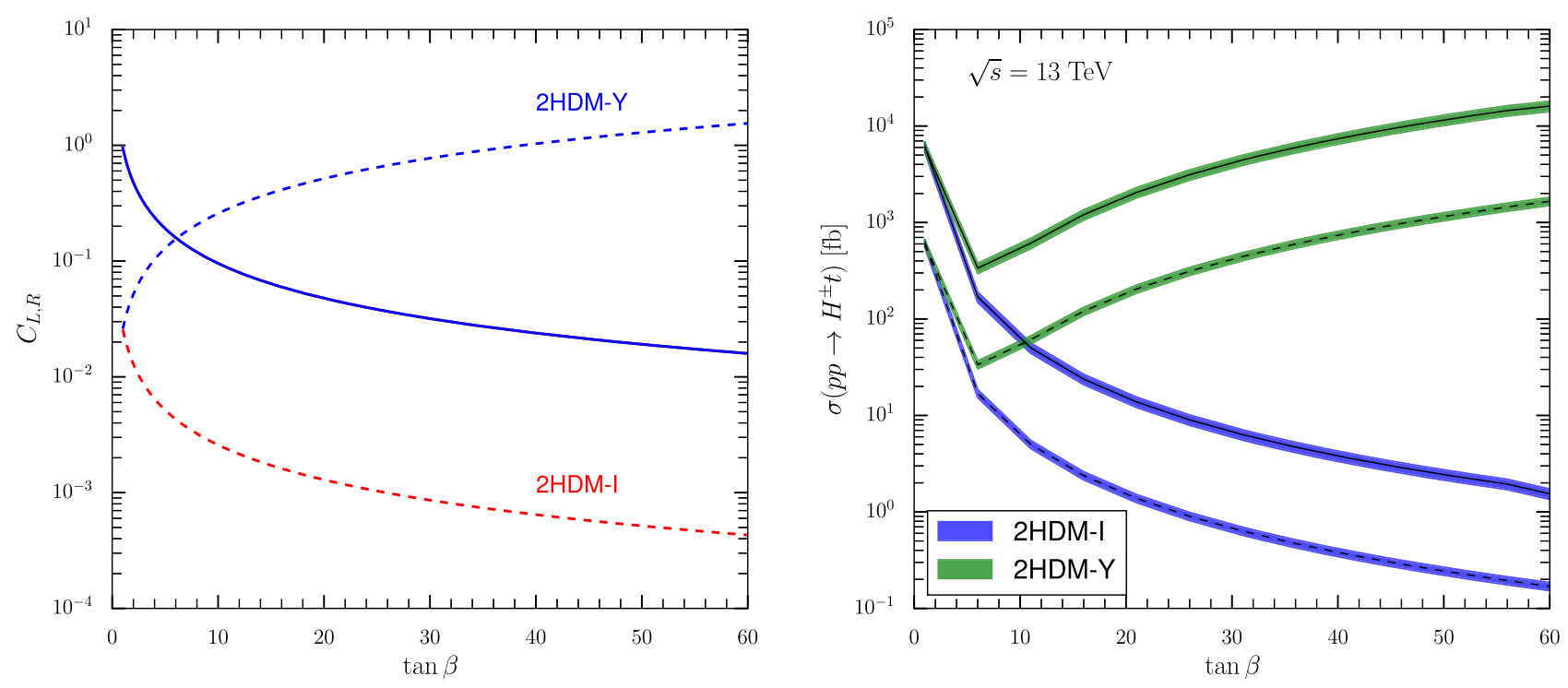

FIG. 2. Left: The chiral form factors of the $H^{+} \bar{t} b$ vertex as a function of $\tan \beta$ for the 2HDM-I (red) and 2HDM-Y (blue), where the overlapping solid(separate dashed) lines show the $\mathrm{L}(\mathrm{R})$-handed components. Right: The $H^{ \pm}$production cross section as a function of $\tan \beta$ in the 2HDM-I (blue) and 2HDM-II (green), where the solid(dashed) lines show the cross sections for $m_{H^{ \pm}}=200 \mathrm{GeV}\left(m_{H^{ \pm}}=500 \mathrm{GeV}\right)$. Here, the error band is a result of the variation of the renormalization/factorization scale by a factor of 2 from the central scale choice defined in Eq. (12) (see below).

\section{C. $t H^{ \pm}$production in the $2 \mathrm{HDM}$}

For the case of a heavy charged Higgs boson, it is appropriate to describe $H^{ \pm}$production in terms of the $b g \rightarrow t H^{-}+$c.c. process [7], so that the cross section is controlled by the $H^{+} \bar{t} b$ coupling. In fact, the decay process $H^{ \pm} \rightarrow t b$ sees the intervention of the same coupling, though the corresponding dependence of the associated BR is somewhat washed away by the fact that this $H^{ \pm}$ decay mode is the dominant one.

The aforementioned vertex can be rewritten in a more convenient form using Eq. (6),

$$
g_{\bar{t} b H^{+}}=i\left(C_{L} P_{L}+C_{R} P_{R}\right),
$$

where $C_{L}=\frac{1}{\sqrt{2} v} m_{t} \kappa_{u}^{A}, C_{R}=\frac{1}{\sqrt{2} v} m_{b} \kappa_{d}^{A}$, wherein $\kappa_{u, d}^{A}$ are given in Table I for the four different types of $2 \mathrm{HDM}$. The nature of the chiral structure of the $H^{+} \bar{t} b$ coupling is strongly dependent on $\tan \beta$. First, in the 2HDM-I (and $2 \mathrm{HDM}-\mathrm{X}$ ), both the R- and L-handed components are proportional to $1 / \tan \beta$ and hence, given that $C_{L} \propto m_{t}$ while $C_{R} \propto m_{b}$, the $H^{+} \bar{t} b$ coupling is dominated by the L-handed component. In contrast, in the 2HDM-II (and 2HDM-Y), the L- and R-handed components of the $H^{+} \bar{t} b$ coupling behave differently depending on $\tan \beta$ : (i) for $\tan ^{2} \beta<m_{t} / m_{b}\left(\tan ^{2} \beta>m_{t} / m_{b}\right)$ the coupling is dominated by the $\mathrm{L}(\mathrm{R})$-handed component; (ii) for $\tan ^{2} \beta \approx m_{t} / m_{b}$, the coupling is purely scalar with no pseudoscalar component.

The dependence of the chiral structure of the $H^{+} \bar{t} b$ vertex and the $H^{ \pm}$production cross section at leading order (LO) as a function of $\tan \beta$ are shown in the left and right panels, respectively, of Fig. 2. ${ }^{1}$ We can see that the cross section in the 2HDM-Y falls to a dip around $\tan ^{2} \beta \approx$ $m_{t} / m_{b}$ and then increases for large $\tan \beta$. In the 2 HDM-I, however, it decreases always for increasing $\tan \beta$. As for the $H^{+} \bar{t} b$ vertex, we notice first that $C_{L}$ is decreasing as a function of $\tan \beta$ in both types of $2 \mathrm{HDM}$ (the corresponding lines in fact overlap). However, in the $2 \mathrm{HDM}-\mathrm{Y}, C_{R}$ can be about three orders of magnitude larger than in the 2HDM-I for large $\tan \beta$. Here, the importance of the L-handed part in the 2 HDM-I through its effect on the $H^{ \pm}$production cross section will induce us to choose $\tan \beta$ as small as possible. Conversely, in the $2 \mathrm{HDM}-\mathrm{Y}$, for large values of $\tan \beta$, the $H^{ \pm}$production cross section can be three times higher than for low $\tan \beta \simeq 1-2$ and the R-handed part of the $H^{+} \bar{t} b$ coupling can be about two orders of magnitudes higher than the L-handed part. Therefore, here, we will choose a large value of $\tan \beta$. Such large $\tan \beta$ is not in conflict with theoretical constraints since one can always tune the Higgs masses and $\mu_{12}^{2}$ parameter for such a purpose.

For definiteness, in what follows, we will choose our benchmark scenarios with $\tan \beta=1$ for the 2HDM-I and $\tan \beta=50$ for the $2 \mathrm{HDM}$-Y, which give

(i) $\left(C_{L}, C_{R}\right)=(0.94,-0.025)$ for 2 HDM-I.

(ii) $\left(C_{L}, C_{R}\right)=(0.019,1.3)$ for $2 \mathrm{HDM}-\mathrm{Y}$.

\footnotetext{
${ }^{1}$ The error bands in the cross section show the effect of the theoretical uncertainties due to factorization/renormalization scale variations. These uncertainties are of order $\pm 14 \%$ for $m_{H^{ \pm}}=$ $200 \mathrm{GeV}$ and decrease to about $\pm 9.7 \%$ for $m_{H^{ \pm}}=500 \mathrm{GeV}$.
} 
We then study three charged Higgs boson masses: $m_{H^{ \pm}}=300,400$ and $500 \mathrm{GeV}$ for $2 \mathrm{HDM}$-I plus $m_{H^{ \pm}}=$ 500, 600 and $700 \mathrm{GeV}$ for $2 \mathrm{HDM}-\mathrm{Y}$.

Before proceeding with our analysis, we should discuss the effects of next-to-LO (NLO) corrections. NLO QCD corrections to the production of a heavy charged Higgs boson in association with a top quark are well known [34-37]. It was found that the corrections can imply a $K$-factor of about 1.3-1.6 to the total production cross section. In Ref. [37], a comprehensive analysis of NLO plus parton shower (PS) effects on different kinematical quantities have been carried in both the 4-flavor scheme (4FS) and 5-flavor scheme (5FS). (Notice that the $g g \rightarrow \bar{b} t H^{-}$ process is the 4FS equivalent of the $b g \rightarrow t H^{-}$channel in the 5FS [38].) Corrections to the $b$-jet transverse momentum give rise to almost a constant $K$-factor over the full $p_{T}$ range. Furthermore, NLO + PS terms improve the agreement between the two schemes especially at large momentum. A very detailed analysis of NLO corrections on the observables we consider in this paper has been carried by the authors of [39]. It was found that angular observables do not receive any substantial corrections. This can be understood by the fact that top quark polarization is parityviolating while $\mathrm{QCD}$ is of a vector nature. In contrast, observables which are based on $b$-jet energy receive some corrections which, however, do not change the shape of the correlated distributions but only their normalization. These findings motivated the use of some forward-backward asymmetries constructed from energy-based observables since they will cancel any possible effects from NLO QCD. The only case where QCD corrections might change the shape of the LO predictions is via nonfactorizable effects (i.e., those which cannot be attributed to either the production or decay stage). However, such corrections are more likely to vanish when the top quark is produced on-shell. Altogether then, we do not expect NLO QCD effects to affect substantially our analysis, apart from an overall $K$-factor, that we ignore here as we are primarily interested in highlighting the fact that is the chiral structure of the $H^{+} \bar{t} b$ vertex enabling one to distinguish between different $2 \mathrm{HDM}$ realizations, rather than the actual normalizations.

\section{PHENOMENOLOGICAL SETUP}

We discuss here our analysis setup.

\section{A. Observables}

As discussed in Sec. II, $\tan \beta$ and $m_{H^{ \pm}}$are the main parameters controlling the production of a charged Higgs boson in association with a top quark and the spin properties of the top quark in this process. The former affects directly the chiral structure of the produced top quark while the latter controls its kinematics. In this section, we discuss the observables that we have used in this study to quantify the sensitivity of this production process to top polarization effects, as function of $\tan \beta$ and $m_{H^{ \pm}}$, in turn enabling one to pursue two goals: (i) to distinguish the charged Higgs boson signal from the SM background, (ii) disentangle different Yukawa types of the 2HDM from each other.

As previously explained, the properties of top (anti) quark emerging at the production level are transmitted to its decays product, i.e., such a quark state decays before hadronizing. Hence, one can study the differential distribution in $\cos \theta_{\ell}^{a}$ of the emerging lepton,

$$
\frac{1}{\sigma} \frac{\mathrm{d} \sigma}{\mathrm{d} \cos \theta_{\ell}^{a}}=\frac{1}{2}\left(1+\alpha_{\ell^{ \pm}} P_{t, \bar{t}} \cos \theta_{\ell}^{a}\right),
$$

wherein $\alpha_{\ell^{ \pm}}$is the so-called spin analyzing power of the charged lepton and $\theta_{\ell^{a}}=\measuredangle\left(\hat{\ell}^{ \pm}, \hat{S}_{a}\right)$, with $\hat{\ell}^{ \pm}$being the direction of flight of the charged lepton in the top quark rest frame and $\hat{S}_{a}$ the spin quantization axis in the basis $a$. There are three such bases relevant for top quark physics at the LHC (see, e.g., [40] for details about the sensitivity and [41] for a corresponding measurement in $t \bar{t}$ production): the helicity basis, the transverse basis and the $r$-basis. In the helicity basis, $\hat{S}_{a}$ is the direction of motion of the top quark in the so-called $(t \bar{t})$ zero momentum frame (ZMF). This basis will be denoted by the superscript $a=k$. In the transverse basis, the spin quantization axis is defined to be orthogonal to the production plane spanned by the (anti)top quark and the beam axis. Finally, the spin quantization axis in the $r$-basis is defined to be orthogonal to those in the helicity and transverse bases. The transverse and $r$-bases, although very useful for anomalous chromomagnetic and chromoelectric top quark coupling studies (see, e.g., [40]), are not a very sensitive probe in the process we are considering here. Therefore, we limit this study to the helicity basis.

It was found that, in addition to angular observables, also energy distributions of the decay products of the (anti)top quark in the laboratory frame and their combination are excellent probes of top quark polarization. This is due to the fact that they acquire a dependence on $\cos \theta_{X}$ (where $X$ is a label of a decay product of the top quark) from boost factors, i.e., factors which bring the decay product from the top quark rest frame to the ( $p p)$ Center-of-Mass (CM) frame. Furthermore, they are sensitive to both the production and decay stages of the top quark. The first two observables are related to the $b$-jet energy in the laboratory frame,

$$
u=\frac{E_{\ell}}{E_{\ell}+E_{b}}, \quad z=\frac{E_{b}}{E_{t}},
$$

where $E_{\ell}, E_{b}$ and $E_{t}$ are the energies of the charged lepton, $b$-jet and top quark in the CM frame, respectively. These observables were studied by the authors of [39] to distinguish $t W^{ \pm}$from $t H^{ \pm}$production. Then, they were 
supplemented by another observable for the purpose of probing top quark $W^{+} \bar{t} b$ anomalous couplings in general [42] and specifically in single top production through the $t$-channel [43]. This is constructed as

$$
x_{\ell}=\frac{2 E_{\ell}}{m_{t}},
$$

where $E_{\ell}$ is the energy of the charged lepton in the CM frame and $m_{t}$ is the (pole) mass of the top quark.

\section{B. Monte Carlo (MC) event generation}

Events are generated here at LO using MADGRAPH5 $\mathrm{AMC} @ \mathrm{NLO}[44]^{2}$ with the NNPDF3.0 parton distribution function (PDF) set using $\alpha_{s}\left(M_{Z}^{2}\right)=0.118$ [45] for both the signal and SM background. The generated events were decayed with MADSPIN [46] to keep full spin correlations between the two produced top (anti)quarks and between each top (anti)quark and its decay products. For the EW parameters, we have used the $G_{\mu}$-scheme in which the input parameters are $G_{F}, \alpha_{\mathrm{em}}$ and $m_{Z^{0}}$. From these parameters, the values of $m_{W^{ \pm}}$and $\sin ^{2} \theta_{W}$ are computed. To this end, we have used the following numerical inputs: $G_{F}=1.16639 \times 10^{-5} \mathrm{GeV}^{-2}, \alpha_{\mathrm{em}}^{-1}(0)=137$ and $m_{Z^{0}}=$ $91.188 \mathrm{GeV}$. For the fermion (pole) masses, we have $m_{t}=$ $172.5 \mathrm{GeV}$ and $m_{b}=4.75 \mathrm{GeV}$.

The decayed events are then passed to PytHIA8 [47] to add PS and hadronization. We have further used the package RIVET [48] for a particle level analysis. Jets are clustered using FASTJET [49].

Finally, we have used a dynamical choice for the renormalization/factorization scale,

$$
\mu_{R}=\mu_{F}=\frac{1}{2} \sum_{i=1}^{N} \sqrt{m_{i}^{2}+p_{T, i}^{2}},
$$

which is nothing but the transverse mass of the final state divided by 2 .

\section{Top quark reconstruction}

In our analysis, the reconstruction of the top (anti)quark is performed based on the PSEUDOTOP definition [50] used widely by the ATLAS and CMS collaborations. Such a method is known to be resilient against initial state radiation (ISR) that contaminates the event sample. Our analysis uses the RIVET implementation of the CMS measurement of the $t \bar{t}$ differential cross section at $\sqrt{s}=8 \mathrm{TeV}$ [51] with a slight modification on the lepton and jet $p_{T}$ thresholds (see below). Further, in this study, we

\footnotetext{
${ }^{2}$ As intimated, NLO QCD effects are not particularly relevant for studying the features of the top-bottom-charged Higgs vertex, so we have ignored these here, for simplicity, despite the tool enables their implementation.
}

require a dressed lepton with cone radius $R_{\ell \gamma}=0.1$. Two approaches are generally used in the reconstruction: the invariant mass based approach and $\Delta R$ based approach. We use the former, where all jets (including $b$-jets), leptons (not from $\tau$ decays though), photons (to dress the selected leptons if they satisfy $\left.\Delta R_{\ell \gamma}=\sqrt{\left(\eta_{\ell}-\eta_{\gamma}\right)^{2}+\left(\phi_{\ell}-\phi_{\gamma}\right)^{2}}<R_{\ell \gamma}\right)$ and missing (transverse) energy are included. The quantity

$K^{2}=\left(M_{\tilde{t}_{\ell}}-m_{t}\right)^{2}+\left(M_{j_{1} j_{2}}-m_{W^{ \pm}}\right)^{2}+\left(M_{\tilde{t}_{h}}-m_{t}\right)^{2}$

is thus minimized to select the hadronic and leptonic top (anti)quarks. In Eq. (13), $\tilde{t}_{\ell}\left(\tilde{t}_{h}\right)$ is the label of the (anti)top constructed in the leptonic(hadronic) decays from the $W^{ \pm}$ boson decays and jet candidates. The minimization procedure adopted in this study can resolve the ambiguity of selecting jets and leptons correctly, e.g., whether a $b$-jet is coming from a semileptonic or hadronic top pair decay.

\section{RESULTS}

Before we illustrate our results, we first discuss our event selection and signal significance.

\section{A. Event selection}

Events are selected if they contain exactly one isolated charged lepton (electron or muon), at least 4 jets (where at least 2 of these are $b$-tagged) and missing transverse energy (which corresponds to the SM neutrinos from $W^{ \pm}$boson decays). The cut flow for the event selection is depicted in Table II with event rates given for, e.g., $\mathcal{L}=200 \mathrm{fb}^{-1}$. Electrons and muons coming from tau decays are not selected as events with one $\tau$ or more are rejected. We require the presence of one electron(muon) with $p_{T}>30 \mathrm{GeV}\left(p_{T}>27 \mathrm{GeV}\right)$ and $|\eta|<2.5(|\eta|<2.4)$. The missing transverse energy is required to satisfy $E_{T}^{\text {miss }}>20 \mathrm{GeV}$. Jets are clustered using the anti- $k_{T}$ algorithm [52] with jet radius $\Delta R=0.5$ (as in the CMS analysis of [53]). We first require $p_{T}>30 \mathrm{GeV}$ and $|\eta|<2.4$ for all the jets in an event. We then refine our selection criteria by vetoing events which do not have a leading jet with $p_{T}>50 \mathrm{GeV}$. Finally, we select events that contain at least 5 jets where at least 3 of these are $b$-tagged. This set of cuts will be denoted by CUTS1. The acceptance times efficiency $(A \times \epsilon)$ for the signal is $31 \%(40 \%)$ for $m_{H^{ \pm}}=300(700) \mathrm{GeV}$. Two more additional cuts on the $H_{T}$ quantity defined by

$$
H_{T}=\sum_{i \in \mathrm{jets}} p_{T}^{i}
$$

were further imposed. The rationale for this is as follows. Background processes have a low peak in the $H_{T}$ distribution compared to the signals, especially for heavy charged Higgs bosons. Hence, imposing cuts on $H_{T}$ will 
TABLE II. The cut flow for the lepton + jets final state at $\sqrt{s}=13 \mathrm{TeV}$ and $\mathcal{L}=200 \mathrm{fb}^{-1}$ for both the signal (in the 2HDM-I and -Y) and background. The details of the event selection are discussed in the text. Normalization is according to the matched flavor scheme discussed below.

\begin{tabular}{|c|c|c|c|c|c|c|c|}
\hline \multirow[b]{2}{*}{$m_{H^{ \pm}}[\mathrm{GeV}]$} & \multirow[b]{2}{*}{ BACKGROUND } & \multicolumn{3}{|c|}{ 2HDM-I } & \multicolumn{3}{|c|}{ 2HDM-Y } \\
\hline & & 300 & 400 & 500 & 500 & 600 & 700 \\
\hline Initial events & 23493940 & 61247 & 28814 & 14615 & 27693 & 14688 & 8148 \\
\hline Cuts1 & 258508 & 19257 & 10477 & 5766 & 9797 & 5579 & 3277 \\
\hline Cuts2 & 163326 & 10590 & 7581 & 5021 & 8367 & 5203 & 3155 \\
\hline Cuts3 & 24230 & 1657 & 1275 & 966 & 1573 & 1211 & 919 \\
\hline
\end{tabular}

improve significantly (beyond the basic selections) the signal-to-background ratio. We impose $H_{T}>500 \mathrm{GeV}$ denoted by CuTs2 and $H_{T}>1000 \mathrm{GeV}$ denoted by CuTS3. (For example, after the $H_{T}>1000 \mathrm{GeV}$ requirement, $A \times \epsilon$ is reduced to about $2.7 \%(11.3 \%)$ for $m_{H^{ \pm}}=300(700) \mathrm{GeV}$.) We adopt these last two cuts as representative of those that more refined selections may adopt in the actual $H^{ \pm}$signal search, still allowing for the latter being enhanced with respect to the background yet without biasing our MC data samples in the direction of removing completely the SM background (and its spin dependent features), as we want to benchmark the $H^{ \pm}$ signals against it.

\section{B. Signal significance}

For the production of a charged Higgs boson in association with a top quark followed by the $H^{ \pm} \rightarrow t b$ decay, where one top decays hadronically and the other one leptonically, there are many background contributions. The most important ones are the exclusive production of a top (anti)quark pair in association with a $b$-quark (i.e., $t \bar{t} b+$ c.c.), top quark pair production in association with a light jet and $t \bar{t}$ inclusive production. The first one is completely irreducible while the second one might contribute since the associated light jet can be mistagged as a $b$-jet, then, the third background is partially reducible since the production of additional $b$-quarks is possible from the parton shower, notably in $g \rightarrow b \bar{b}$ splitting, but it is not a leading effect. The light- and $c$-jet misidentification probability is about $1 / 150$ and $1 / 4$, respectively, at high $p_{T}$. Therefore, we have generated the $t \bar{t}+$ jet background with a $p_{T}>100 \mathrm{GeV}$ cut on the extra jet. There are further possible background processes, such as single top, diboson and $W+$ jet production, but these are generally negligible compared to the previous ones.

Using the described top (anti)quark reconstruction procedure on both heavy flavor states combined with the requirements on jet activity and the cuts in $H_{T}$ will reduce substantially the background. To enable the possible observation of a signal, we compute its significance defined as [54]

$$
\mathcal{Z}=\sqrt{2\left(\left(N_{s}+N_{b}\right) \log \left(1+\frac{N_{s}}{N_{b}}\right)-N_{s}\right)},
$$

where $N_{s}\left(N_{b}\right)$ is the number of signal(background) events after a given selection. We finally compute $\mathcal{Z}$ for $\mathcal{L}=200$ and $1000 \mathrm{fb}^{-1}$ of integrated LHC luminosity. The obtained values are displayed in Table III. Here, we can see that already the basic selection (including the jet multiplicity requirement) enhances significantly the value of $\mathcal{Z}$. However, due to the small value of the cross sections for $m_{H^{ \pm}}=500 \mathrm{GeV}$ upwards, the cuts on $H_{T}$ are necessary to enhance significantly $\mathcal{Z}$ (at large luminosity). Indeed, we can see that, e.g., after CuTS2, $\mathcal{Z}$ increases by a factor of $\simeq 1.2$ for $m_{H^{ \pm}}=500 \mathrm{GeV}$ while it even decreases for lighter charged Higgs bosons. Before moving on, we should like to remind the reader here that these

TABLE III. Signal significance of the two types of 2HDM that we study for $m_{H^{ \pm}}=300,400$ and $500 \mathrm{GeV}$ in the 2HDM-I and $m_{H^{ \pm}}=500,600$ and $700 \mathrm{GeV}$ in the $2 \mathrm{HDM}$-Y. The numbers outside (inside) the brackets refer to the case of $200(1000) \mathrm{fb}^{-1} \mathrm{of}$ total luminosity. Normalization is according to the matched flavor scheme discussed below.

\begin{tabular}{|c|c|c|c|c|c|c|}
\hline \multirow[b]{3}{*}{$m_{H^{ \pm}}[\mathrm{GeV}]$} & \multicolumn{6}{|c|}{$\mathcal{L}=200(1000) \mathrm{fb}^{-1}$} \\
\hline & \multicolumn{3}{|c|}{ 2HDM-I } & \multicolumn{3}{|c|}{ 2HDM-Y } \\
\hline & 300 & 400 & 500 & 500 & 600 & 700 \\
\hline Initial events & $12.63(28.24)$ & 5.94(13.29) & $3.01(6.74)$ & $5.71(12.77)$ & $3.03(6.77)$ & $1.68(3.75)$ \\
\hline Cuts1 & $37.42(83.32)$ & $20.41(45.36)$ & $11.30(25.32)$ & $19.15(43.14)$ & $10.93(24.68)$ & $6.43(14.31)$ \\
\hline Cuts2 & $25.92(57.67)$ & 18.61(41.47) & $12.36(27.82)$ & $20.53(46.21)$ & $12.80(28.82)$ & 7.78(17.31) \\
\hline Cuts3 & $10.53(22.83)$ & $8.12(17.64)$ & $6.16(13.62)$ & $10.00(22.55)$ & 7.71(17.66) & $5.87(13.26)$ \\
\hline
\end{tabular}


significances should not be regarded as a means of claiming discovery through our selection, rather as a means to indicate how to purify the signal so as to entertain the study of spin dependent observables that we illustrate now.

\section{Results}

We present our results selecting three observables: $\cos \theta_{\ell}^{k}$ (i.e., in the helicity basis), $x_{\ell}$ and $u$. (We neglect showing the $z$ spectra, as they offer far less sensitivity in comparison.) The distributions are shown, after applying both the basic selection cuts (denoted by CUTS1) and the enhanced ones (e.g., CuTs3) in Figs. 3 and 4.
In Fig. 3, we show the $\cos \theta_{\ell}^{k}$ spectrum. The left panel shows the distribution after the CUTS1 set. The label SM shows the irreducible contribution to the background coming from the $t \bar{t} b+$ c.c. process, which is the dominant one, though not the full SM noise (hence the different acronym). We can see clearly that the SM curves exhibit almost no dependence on $\cos \theta_{\ell}^{k}$ except for regions of $\cos \theta_{\ell} \in[-0.75,0]$ where the EW contribution becomes important. This is unsurprising because it is otherwise dominated by a gluon intermediate state. Since this contribution is of vector nature, the polarization of the produced top quark is essentially zero. The interesting
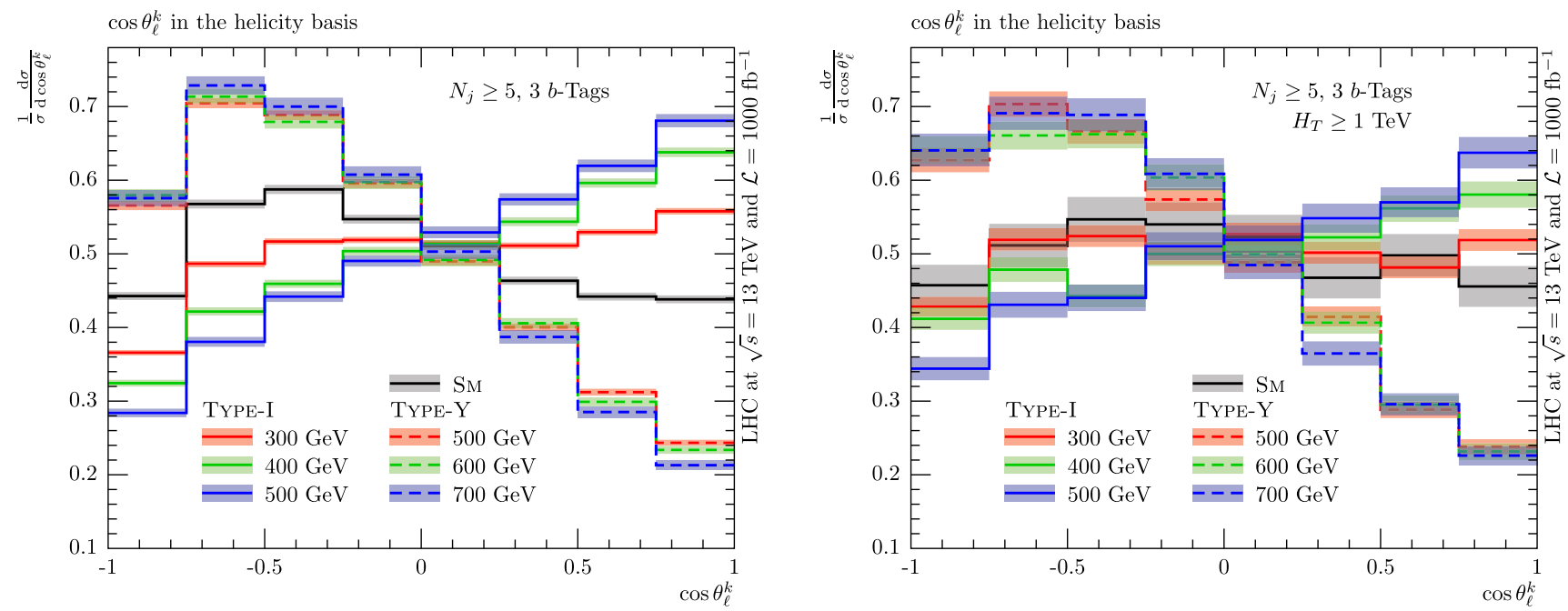

FIG. 3. Left: The $\cos \theta_{\ell}^{k}$ distributions for the SM (solid black), 2HDM-I (solid red, green and blue) and 2HDM-Y (dashed red, green and blue) after applying all the basic selections. Right: the same distribution but for $H_{T}>1 \mathrm{TeV}$. Data are for $\sqrt{s}=13 \mathrm{TeV}$ and $\mathcal{L}=1000 \mathrm{fb}^{-1}$. The color scheme identify different $H^{ \pm}$masses while the shading represents the statistical and luminosity error.
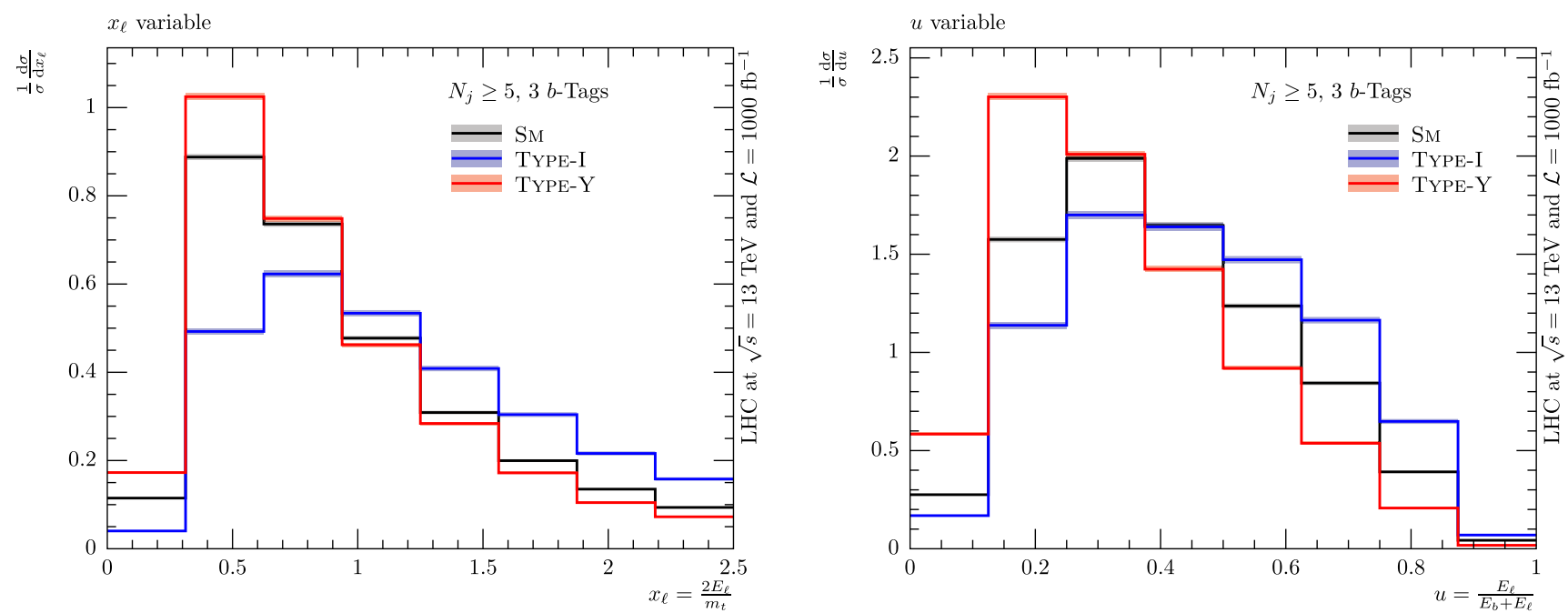

FIG. 4. The $x_{\ell}$ (left panel) and $u$ (right panel) distributions for the SM (black), 2HDM-I (blue) and 2HDM-Y (red) applying the first set of cuts (denoted by CuTS1). Data are for $\sqrt{s}=13 \mathrm{TeV}$ and $\mathcal{L}=1000 \mathrm{fb}^{-1}$. The (almost invisible) shading represents the statistical and luminosity error. Here, $m_{H^{ \pm}}=500 \mathrm{GeV}$. 
observation is that the 2HDM-I (dominated in our benchmark by the L-handed component) and 2HDM-Y (dominated in our benchmark by the R-handed component) have opposite slopes and hence different polarization (with different sign). In the $2 \mathrm{HDM}-\mathrm{Y}$, the polarization of the top quark is negative while in the 2HDM-I it is positive. We remind the reader here that the charged lepton is $100 \%$ correlated with the parent top quark and hence the slope of the $\cos \theta_{\ell}^{k}$ distribution is directly proportional to the top quark polarization. Furthermore, the distribution has a drop for $\cos \theta_{\ell}^{k} \simeq-0.8$ due to the isolation cuts. Finally, we note that, after applying the cut $H_{T}>1000 \mathrm{GeV}$, one can see in the right panel of Fig. 3 that the sensitivity decreases somewhat, especially for $2 \mathrm{HDM}-\mathrm{I}$, though it remains very noticeable.

We should now also comment on the effect of changing the charged Higgs boson mass on the distributions of the angular observable. In the previous section, we have shown that $\tan \beta$ controls the chiral structure of the $H^{+} \bar{t} b$ coupling and hence directly its polarization. However, since the latter is weighted by the cross section (via the helicity amplitudes entering the scattering matrix element and the phase space), we expect that it depends on the mass of the charged Higgs boson as well. We have checked this assumption for various values of the charged Higgs boson mass, i.e., $m_{H^{ \pm}}=300(500), 400(600)$ and $500(700) \mathrm{GeV}$ for the $2 \mathrm{HDM}-\mathrm{I}(2 \mathrm{HDM}-\mathrm{Y})$, and found that this is correct. First of all, for $m_{H^{ \pm}}=200 \mathrm{GeV}$, there is almost no sensitivity to the different chiral structures of the 2HDM-I and 2HDM-Y, as both models give approximately the same predictions and cannot be distinguished from the SM either. (This is why we do not show this case.) However, starting from $m_{H^{ \pm}}=300 \mathrm{GeV}$, the studied observables start to exhibit the aforementioned differences between the 2HDM-I, 2HDM-Y and SM with a maximum sensitivity at large mass, e.g., $m_{H^{ \pm}}=500 \mathrm{GeV}$ for the 2HDM-I while for the $2 \mathrm{HDM}-\mathrm{Y}$ the charged Higgs mass dependence is essentially negligible. Henceforth, then, we shall adopt $m_{H^{ \pm}}=500 \mathrm{GeV}$ as reference charged Higgs mass value for our two BSM scenarios.
In Fig. 4, we show the $x_{\ell}$ (left panel) and $u$ (right panel) spectra. The $x_{\ell}$ distribution shows no differences between the SM and 2HDM-Y and this is due to cancellations among different terms proportional to the sign of the polarization, while for the 2HDM-I there are two discernible features: the position of the peak (which is slightly shifted respect to the SM) and the behavior in the tail of the distribution. In contrast, $u$ is more sensitive and has a higher separation power than $x_{\ell}$ across the three theoretical setups, especially for the regions $0<u<0.25$ and $0.5<u<0.85$. In both these cases, the three scenarios are clearly distinguishable. Not shown here, we report that the effects of cuts on $H_{T}$ diminish somehow the sensitivity of both the $x_{\ell}$ and $u$ distribution to the underlying model assumption.

\section{Asymmetries}

After showing the potential of the spin observables to distinguish between the SM and the different Yukawa realizations of the $2 \mathrm{HDM}$, an interesting question is how to minimize the effect of systematic effects, of both theoretical (e.g., PDFs, missing higher order corrections, etc.) and experimental (i.e., detector mis-behavior) origin, that would affect these (and that we cannot emulate here). For this purpose, we suggest the use of forward-backward asymmetries constructed from the observables defined in Eqs. (9)-(10) and displayed in Figs. 3-4. Asymmetries are further resilient to NLO QCD corrections. We define an asymmetry $A_{X}$ as

$$
A_{X}=\frac{\sigma\left(X>X_{c}\right)-\sigma\left(X<X_{c}\right)}{\sigma\left(X>X_{c}\right)+\sigma\left(X<X_{c}\right)},
$$

with $X=\cos \theta_{\ell}^{k}, x_{\ell}, u$ and where $X_{c}$ is a reference point for the asymmetry $A_{X}$. In the present study, we choose the following references points: $\cos \theta_{\ell, c}^{k}=0, x_{\ell, c}=0.6$ and $u_{c}=0.5$. In Table IV, we show the values of the three asymmetries in the SM and the two usual $2 \mathrm{HDM}$ types. It is clear that $A_{\theta_{\ell}}$ can distinguish between the SM and the two realizations of the $2 \mathrm{HDM}$ considered here. Furthermore, this asymmetry can even distinguish between the different

TABLE IV. The asymmetries $A_{\theta_{f}}, A_{x_{\ell}}$ and $A_{u}$ for the SM background, 2HDM-I $\left(m_{H^{ \pm}}=300,400\right.$ and 500 GeV) and 2HDM-Y $\left(m_{H^{ \pm}}=500,600\right.$ and $\left.700 \mathrm{GeV}\right)$. For each asymmetry, the first row corresponds to the values computed after basic selections (denoted in the text by CUTS1) while the second row corresponds to the values for $H_{T}>1000 \mathrm{GeV}$ (denoted by CuTS1). The quoted errors for each asymmetry correspond to statistical uncertainties estimated as $\Delta A_{X}=\sqrt{1-A_{X}^{2}} / \sqrt{N}$ [42].

\begin{tabular}{|c|c|c|c|c|c|c|c|}
\hline \multirow[b]{2}{*}{ Asymmetry } & \multirow[b]{2}{*}{ BACKGROUND } & \multicolumn{3}{|c|}{ 2HDM-I } & \multicolumn{3}{|c|}{ 2HDM-Y } \\
\hline & & $300 \mathrm{GeV}$ & $400 \mathrm{GeV}$ & $500 \mathrm{GeV}$ & $500 \mathrm{GeV}$ & $600 \mathrm{GeV}$ & $700 \mathrm{GeV}$ \\
\hline$A_{\theta_{\ell}}$ & $\begin{array}{l}-0.04 \pm 0.001 \\
-0.01 \pm 0.003\end{array}$ & $\begin{array}{l}0.05 \pm 0.003 \\
0.01 \pm 0.014\end{array}$ & $\begin{array}{l}0.14 \pm 0.004 \\
0.08 \pm 0.012\end{array}$ & $\begin{array}{l}0.20 \pm 0.005 \\
0.13 \pm 0.013\end{array}$ & $\begin{array}{l}-0.27 \pm 0.004 \\
-0.28 \pm 0.009\end{array}$ & $\begin{array}{l}-0.28 \pm 0.005 \\
-0.28 \pm 0.011\end{array}$ & $\begin{array}{l}-0.31 \pm 0.007 \\
-0.31 \pm 0.013\end{array}$ \\
\hline$A_{x_{\ell}}$. & $\begin{array}{l}0.37 \pm 0.001 \\
0.54 \pm 0.003\end{array}$ & $\begin{array}{l}0.40 \pm 0.003 \\
0.53 \pm 0.008\end{array}$ & $\begin{array}{l}0.52 \pm 0.003 \\
0.57 \pm 0.009\end{array}$ & $\begin{array}{l}0.65 \pm 0.004 \\
0.65 \pm 0.010\end{array}$ & $\begin{array}{l}0.21 \pm 0.004 \\
0.30 \pm 0.009\end{array}$ & $\begin{array}{l}0.27 \pm 0.005 \\
0.33 \pm 0.010\end{array}$ & $\begin{array}{l}0.33 \pm 0.007 \\
0.38 \pm 0.012\end{array}$ \\
\hline$A_{u}$ & $\begin{array}{l}-0.35 \pm 0.001 \\
-0.35 \pm 0.003\end{array}$ & $\begin{array}{l}-0.30 \pm 0.003 \\
-0.27 \pm 0.009\end{array}$ & $\begin{array}{l}-0.22 \pm 0.004 \\
-0.31 \pm 0.011\end{array}$ & $\begin{array}{l}-0.16 \pm 0.005 \\
-0.26 \pm 0.012\end{array}$ & $\begin{array}{l}-0.58 \pm 0.003 \\
-0.63 \pm 0.008\end{array}$ & $\begin{array}{l}-0.58 \pm 0.004 \\
-0.64 \pm 0.009\end{array}$ & $\begin{array}{l}-0.58 \pm 0.006 \\
-0.62 \pm 0.010\end{array}$ \\
\hline
\end{tabular}



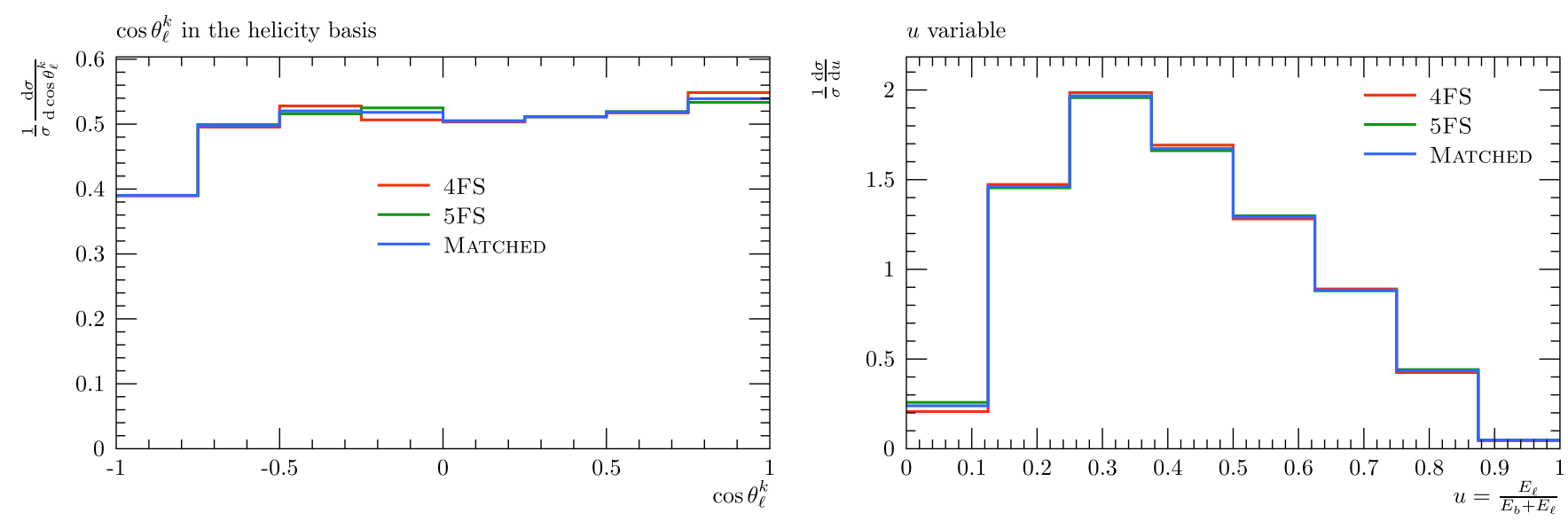

FIG. 5. Left: The normalized distribution for the $\cos \theta_{\ell}^{k}$ variable. Right: The normalized distribution for the $u$ variable. The two distributions are shown for $m_{H^{ \pm}}=300 \mathrm{GeV}$ and $\tan \beta=1$ after passing the CUTS2 requirements. In both panels, predictions are shown for the 4FS (red), 5FS (green) and matched scheme (blue).

masses for the $2 \mathrm{HDM}-\mathrm{I}$ case. However, for the $2 \mathrm{HDM}-\mathrm{Y}$, $A_{\theta_{\ell}}$ is insensitive to the mass of the charged Higgs boson. However, $A_{x_{\ell}}$ is able to remove this degeneracy for the 2HDM-Y.

\section{E. Effects of matching}

As previously mentioned, the rate of both inclusive and exclusive $p p \rightarrow t H^{ \pm}+X$ production can be estimated in two different flavor schemes: 4FS and, like we have done here, 5FS. In fact, as either of the 4FS and 5FS approach separately misses relevant features present in the other, the best approach is matching the two schemes, by adding the two processes and subtracting the common terms. At any rate, we need to establish whether our results are sensitive to the choice of flavor content of the proton. For this reason, we discuss, in what follows, how differences due to the different schemes impact on the observables we have studied throughout this paper. In the 4FS, $t H^{-}$is produced with an extra $b$-antiquark while, in the 5FS, the $b$-quark is treated as a massless parton inside the proton which PDF is estimated by solving the relevant Dokshitzer-GribovLipatov-Altarelli-Parisi (DGLAP) equations [55]. In the 4FS, the cross section is smaller than in the 5FS due to both the presence of the extra $b$-antiquark in the final state, implying a phase space suppression, and the fact that the $b$-quark PDF includes additional logarithmic corrections with respect to the gluon one. Specifically, for the charged Higgs production scenarios considered in this study, the ratio $\sigma_{5 \mathrm{FS}} / \sigma_{4 \mathrm{FS}} \simeq 2$, as estimated at the scale defined in Eq. (12). ${ }^{3}$ Therefore, as intimated, a perturbatively more reliable way to get an estimate of the charged Higgs boson

\footnotetext{
${ }^{3}$ For reference, notice that, for SM Higgs boson production, the cross section in the 4FS is a factor of 5 smaller than in the 5FS for the factorization/renormalization scale choice $\mu_{F} \equiv \mu_{R}=m_{H_{\mathrm{SM}}}$.
}

production rate is to use a matching procedure. We use here the so-called Santander matching (which details can be found in, e.g., Ref. [56]) to combine cross sections in the two schemes. Herein, the total cross section can be written as

$$
\sigma_{\text {matched }}=\frac{\sigma_{4 \mathrm{FS}}+\omega \sigma_{5 \mathrm{FS}}}{1+\omega}
$$

where $\omega=\log \left(m_{H^{ \pm}} / m_{b}\right)-2$. It is then clear that the 4FS (5FS) dominates for very small(large) logarithms. For the benchmark points that we have chosen in our paper, the $\omega$ factor takes values of about 2.15-3. However, the key point here is to assess the effects of the choice of flavor scheme (4FS, 5FS or matched case) on the observables we studied in this paper. To this end, we adopt here as an example one of the benchmark scenarios studied, i.e., the 2HDM-I with $m_{H^{ \pm}}=300 \mathrm{GeV}$. In Fig. 5, we show the two key distributions that can distinguish between 2HDM types, i.e., $\cos \theta_{\ell}^{k}$ and $u$, as they appear in the 4FS, 5FS and the matched case. As we can see from this figure, there is no difference between the three cases. This holds true for all spin observables, in fact, including the asymmetries, not shown here, independently of the event selection setup. Likewise, no significant differences amongst the three flavor schemes emerge in the other $2 \mathrm{HDM}$ configurations either.

\section{CONCLUSION}

In summary, in the pursuit to establish a heavy charged Higgs boson signal at the LHC via its $H^{ \pm} \rightarrow t b$ decays, we have shown that spin dependent observables, both angles and energy fractions of the (anti)top decay products, may improve the sensitivity of current analyses for the purpose of both $H^{ \pm}$discovery and characterization, as they lead to forward-backward asymmetries that are clearly different in 
two types of $2 \mathrm{HDM}$ from the SM and from each other. We have proven this to be true for the case of two benchmark points over the parameter spaces of the 2HDM-I and 2HDM-Y, crucially differing in the chiral structure of the $H^{+} \bar{t} b$ vertex. Further, we have shown that such differences persist irrespectively of the $H^{ \pm}$mass value, so long that the latter is $300 \mathrm{GeV}$ or more. Hence, just like the exploitation of spin effects has been fruitful over many years for the case of $H^{ \pm} \rightarrow \tau \nu$ decays (based on the pioneering work of Ref. [57]), we advocate a similar approach in the case of $H^{ \pm} \rightarrow t b$ decays to now be taken.

We are confident that this will improve the LHC sensitivity to heavy charged bosons as we have reached these conclusions by exploiting rather sophisticated phenomenological tools, adopting exact matrix element estimates, parton shower dynamics, hadronization effects as well as jet definitions mimicking closely a typical experimental setup at the LHC, albeit we have not vigorously pursued a fully-fledged signal-to-background selection. We would expect this to be attempted now by ATLAS and CMS, possibly in conjunction with multivariate analysis methods trained to learn the different spin dynamics affecting an $H^{ \pm}$induced signal and a $W^{ \pm}$dominated background. In fact, the illustrated spin dynamics is rather robust against higher order QCD corrections as well as the choice of flavor scheme inside the proton.

Finally, we should close by remarking that, while we have illustrated all of the above by adopting two specific paradigms for BSM physics containing $H^{ \pm}$ bosons, our approach can be exploited for a variety of other new physics frameworks containing such (pseudo) scalar charged states (e.g., the Georgi-Machacek scenario, LR models, Supersymmetry, etc.), so long that they induce chiral structures in the $H^{+} \bar{t} b$ vertex that are predominantly L- or R-handed.

\section{ACKNOWLEDGMENTS}

The authors would like to thank Marco A. Harrendorf for his collaboration in the early stage of this work. The work of A. J. was supported by Shanghai Pujiang Program. S. M. is supported in part through the NExT Institute and the STFC CG ST/L000296/1. A. A. and S.M. acknowledge funding via the H2020-MSCA-RISE-2014 Grant No. 645722 (NonMinimalHiggs). A. A. acknowledges the Alexander von Humboldt Foundation and the Max Planck Institute for Physics, Munich, where part of this work has been done.
[1] S. Abachi et al. (D0 Collaboration), Observation of the Top Quark, Phys. Rev. Lett. 74, 2632 (1995).

[2] F. Abe et al. (CDF Collaboration), Observation of Top Quark Production in $\bar{p} p$ Collisions, Phys. Rev. Lett. 74, 2626 (1995).

[3] W. Bernreuther, Top quark physics at the LHC, J. Phys. G 35, 083001 (2008).

[4] F. Dliot, N. Hadley, S. Parke, and T. Schwarz, Properties of the top quark, Annu. Rev. Nucl. Part. Sci. 64, 363 (2014).

[5] W. Bernreuther and P. Uwer, Top-quark physics at colliders, Nucl. Part. Phys. Proc. 261-262, 414 (2015).

[6] G. C. Branco, P. M. Ferreira, L. Lavoura, M. N. Rebelo, M. Sher, and J. P. Silva, Theory and phenomenology of twoHiggs-doublet models, Phys. Rep. 516, 1 (2012).

[7] A. G. Akeroyd et al., Prospects for charged Higgs searches at the LHC, Eur. Phys. J. C 77, 276 (2017).

[8] A. Stange and S. Willenbrock, Yukawa correction to top quark production at the Tevatron, Phys. Rev. D 48, 2054 (1993).

[9] H.-Y. Zhou, C.-S. Li, and Y.-P. Kuang, Yukawa corrections to top quark production at the LHC in two Higgs doublet models, Phys. Rev. D 55, 4412 (1997).

[10] W. Hollik, W. M. Mosle, and D. Wackeroth, Top pair production at hadron colliders in nonminimal standard models, Nucl. Phys. B516, 29 (1998).
[11] A. Denner and A. H. Hoang, The top decay $t \rightarrow b W$ in the two Higgs doublet model, Nucl. Phys. B397, 483 (1993).

[12] W. Bernreuther, P. Gonzalez, and M. Wiebusch, The top quark decay vertex in standard model extensions, Eur. Phys. J. C 60, 197 (2009).

[13] K. Huitu, S. K. Rai, K. Rao, S. D. Rindani, and P. Sharma, Probing top charged-Higgs production using top polarization at the large hadron collider, J. High Energy Phys. 04 (2011) 026.

[14] A. Arhrib and A. Jueid, $t b W$ anomalous couplings in the two Higgs doublet model, J. High Energy Phys. 08 (2016) 082.

[15] G. Eilam, J. L. Hewett, and A. Soni, Rare decays of the top quark in the standard and two Higgs doublet models, Phys. Rev. D 44, 1473 (1991); Erratum, Phys. Rev. D 59, 039901(E) (1998).

[16] G. Mahlon and S. J. Parke, Angular correlations in top quark pair production and decay at hadron colliders, Phys. Rev. D 53, 4886 (1996).

[17] G. Mahlon and S. J. Parke, Spin correlation effects in top quark pair production at the LHC, Phys. Rev. D 81, 074024 (2010).

[18] P. Uwer, Maximizing the spin correlation of top quark pairs produced at the Large Hadron Collider, Phys. Lett. B 609, 271 (2005). 
[19] S. L. Glashow and S. Weinberg, Natural conservation laws for neutral currents, Phys. Rev. D 15, 1958 (1977).

[20] M. Aoki, S. Kanemura, K. Tsumura, and K. Yagyu, Models of Yukawa interaction in the two Higgs doublet model, and their collider phenomenology, Phys. Rev. D 80, 015017 (2009).

[21] J. F. Gunion and H. E. Haber, The $C P$ conserving two Higgs doublet model: The approach to the decoupling limit, Phys. Rev. D 67, 075019 (2003).

[22] M. Misiak and M. Steinhauser, Weak radiative decays of the B meson and bounds on $M_{H^{ \pm}}$in the two-Higgs-doublet model, Eur. Phys. J. C 77, 201 (2017).

[23] A. Arhrib, R. Benbrik, S. Moretti, R. Santos, and P. Sharma, Signal to background interference in $p p \rightarrow t H^{-} \rightarrow t W^{-} b \bar{b}$ at the LHC Run-II, Phys. Rev. D 97, 075037 (2018).

[24] S. Schael et al. (DELPHI, OPAL, and ALEPH Collaborations, LEP Working Group for Higgs Boson Searches, L3 Collaboration), Search for neutral MSSM Higgs bosons at LEP, Eur. Phys. J. C 47, 547 (2006).

[25] G. Aad et al. (ATLAS Collaboration), Search for charged Higgs bosons decaying via $H^{ \pm} \rightarrow \tau^{ \pm} \nu$ in fully hadronic final states using $p p$ collision data at $\sqrt{s}=8 \mathrm{TeV}$ with the ATLAS detector, J. High Energy Phys. 03 (2015) 088.

[26] V. Khachatryan et al. (CMS Collaboration), Search for a light charged Higgs boson decaying to $\mathrm{c} \overline{\mathrm{s}}$ in $\mathrm{pp}$ collisions at $\sqrt{s}=8 \mathrm{TeV}$, J. High Energy Phys. 12 (2015) 178.

[27] A. Arhrib, R. Benbrik, and S. Moretti, Bosonic decays of charged Higgs bosons in a 2HDM type-I, Eur. Phys. J. C 77, 621 (2017).

[28] A. Arhrib, R. Benbrik, H. Harouiz, S. Moretti, and A. Rouchad, A guidebook to hunting charged Higgs bosons at the LHC, arXiv:1810.09106.

[29] A. Arhrib, K. Cheung, J. S. Lee, and C.-T. Lu, Enhanced charged Higgs production through W-Higgs fusion in W-b scattering, J. High Energy Phys. 05 (2016) 093.

[30] A. Arbey, F. Mahmoudi, O. Stal, and T. Stefaniak, Status of the charged Higgs boson in two Higgs doublet models, Eur. Phys. J. C 78, 182 (2018).

[31] D. Eriksson, J. Rathsman, and O. Stal, 2HDMC: TwoHiggs-doublet model calculator physics and manual, Comput. Phys. Commun. 181, 189 (2010).

[32] P. Bechtle, S. Heinemeyer, O. Stal, T. Stefaniak, and G. Weiglein, Higgs Signals: Confronting arbitrary Higgs sectors with measurements at the tevatron and the LHC, Eur. Phys. J. C 74, 2711 (2014).

[33] P. Bechtle, O. Brein, S. Heinemeyer, O. Stal, T. Stefaniak, G. Weiglein, and K. E. Williams, HiggsBounds - 4: Improved tests of extended Higgs sectors against exclusion bounds from LEP, the tevatron and the LHC, Eur. Phys. J. C 74, 2693 (2014).

[34] S.-h. Zhu, Complete next-to-leading order QCD corrections to charged Higgs boson associated production with top quark at the CERN large hadron collider, Phys. Rev. D 67, 075006 (2003).

[35] E. L. Berger, T. Han, J. Jiang, and T. Plehn, Associated production of a top quark and a charged Higgs boson, Phys. Rev. D 71, 115012 (2005).

[36] M. Klasen, K. Kovarik, P. Nason, and C. Weydert, Associated production of charged Higgs bosons and top quarks with POWHEG, Eur. Phys. J. C 72, 2088 (2012).
[37] C. Degrande, M. Ubiali, M. Wiesemann, and M. Zaro, Heavy charged Higgs boson production at the LHC, J. High Energy Phys. 10 (2015) 145.

[38] M. Guchait and S. Moretti, Improving the discovery potential of charged Higgs bosons at tevatron run II, J. High Energy Phys. 01 (2002) 001.

[39] R. M. Godbole, L. Hartgring, I. Niessen, and C. D. White, Top polarisation studies in $H^{-} t$ and $W t$ production, J. High Energy Phys. 01 (2012) 011.

[40] W. Bernreuther and Z.-G. Si, Top quark spin correlations and polarization at the LHC: Standard Model predictions and effects of anomalous top chromo moments, Phys. Lett. B 725, 115 (2013); Erratum, Phys. Lett. B 744, 413 (E) (2015).

[41] M. Aaboud et al. (ATLAS Collaboration), Measurements of top quark spin observables in $t \bar{t}$ events using dilepton final states in $\sqrt{s}=8 \mathrm{TeV}$ pp collisions with the ATLAS detector, J. High Energy Phys. 03 (2017) 113.

[42] V. A. Prasath, R. M. Godbole, and S. D. Rindani, Longitudinal top polarisation measurement and anomalous $W t b$ coupling, Eur. Phys. J. C 75, 402 (2015).

[43] A. Jueid, Probing anomalous Wtb couplings at the LHC in single $t$-channel top quark production, Phys. Rev. D 98, 053006 (2018).

[44] J. Alwall, M. Herquet, F. Maltoni, O. Mattelaer, and T. Stelzer, MadGraph 5: Going beyond, J. High Energy Phys. 06 (2011) 128.

[45] R. D. Ball et al. (NNPDF Collaboration), Parton distributions for the LHC Run II, J. High Energy Phys. 04 (2015) 040 .

[46] P. Artoisenet, R. Frederix, O. Mattelaer, and R. Rietkerk, Automatic spin-entangled decays of heavy resonances in Monte Carlo simulations, J. High Energy Phys. 03 (2013) 015 .

[47] T. Sjöstrand, S. Ask, J. R. Christiansen, R. Corke, N. Desai, P. Ilten, S. Mrenna, S. Prestel, C. O. Rasmussen, and P.Z. Skands, An introduction to PYTHIA 8.2, Comput. Phys. Commun. 191, 159 (2015).

[48] A. Buckley, J. Butterworth, L. Lonnblad, D. Grellscheid, H. Hoeth, J. Monk, H. Schulz, and F. Siegert, Rivet user manual, Comput. Phys. Commun. 184, 2803 (2013).

[49] M. Cacciari, G. P. Salam, and G. Soyez, Fastjet user manual, Eur. Phys. J. C 72, 1896 (2012).

[50] (CMS Collaboration), Object definitions for top quark analyses at the particle level, Technical Report No. CMSNOTE-2017-004. CERN-CMS-NOTE-2017-004, CERN, Geneva, 2017.

[51] V. Khachatryan et al. (CMS Collaboration), Measurement of the differential cross section for top quark pair production in pp collisions at $\sqrt{s}=8 \mathrm{TeV}$, Eur. Phys. J. C 75, 542 (2015).

[52] M. Cacciari, G. P. Salam, and G. Soyez, The anti-k(t) jet clustering algorithm, J. High Energy Phys. 04 (2008) 063.

[53] V. Khachatryan et al. (CMS Collaboration), Search for a charged Higgs boson in pp collisions at $\sqrt{s}=8 \mathrm{TeV}$, J. High Energy Phys. 11 (2015) 018.

[54] G. Cowan, K. Cranmer, E. Gross, and O. Vitells, Asymptotic formulae for likelihood-based tests of new physics, 
Eur. Phys. J. C 71, 1554 (2011); Erratum, Eur. Phys. J. C 73, 2501(E) (2013).

[55] R. M. Barnett, H. E. Haber, and D. E. Soper, Ultraheavy particle production from heavy partons at hadron colliders, Nucl. Phys. B306, 697 (1988).
[56] R. Harlander, M. Kramer, and M. Schumacher, Bottomquark associated Higgs-boson production: Reconciling the four- and five-flavour scheme approach, arXiv:1112.3478.

[57] D. P. Roy, Using $\tau$ polarization for the charged Higgs search at hadron colliders, Phys. Lett. B 277, 183 (1992). 